\title{
Design of Broadband Dual-Frequency Microstrip Patch Antenna with Modified Sierpinski Fractal Geometry
}

\author{
Ngu Sze Song, Kho Lee Chin \\ Electronics Engineering, Faculty of Engineering \\ Universiti Malaysia Sarawak (UNIMAS) \\ Sarawak, Malaysia \\ ssngu@feng.unimas.my
}

\author{
David Bong Boon Liang, Martin Anyi \\ Electronics Engineering, Faculty of Engineering \\ Universiti Malaysia Sarawak (UNIMAS) \\ Sarawak, Malaysia. \\ lckho@feng.unimas.my
}

\begin{abstract}
Fractal antennas have the characteristic of radiating in multiple frequencies through the property of self similarity that fractal shapes posses. By connecting fractal shaped antennas, wideband coverage can be achieved. Microstrip patch antennas with Sierpinski fractal geometry can be tuned, by design, to work exactly at the bands of interest, through judicious choice of the fractal designs and iteration. Therefore, a broadband dualfrequency microstip patch antenna with modified Sierpienski fractal geometry is designed by using Microwave Office 2002 simulation software. The broadband and multiple frequency characteristics of fractal antennas will be demonstrated. The performance of microstrip patch antenna with the classic and modified Sierpinski fractal geometries will be presented.
\end{abstract}

Keywords-fractal antenna; dual-frequency band; microstrip patch antenna

\section{INTRODUCTION}

Antenna is a specialized transducer that converts radio frequency (RF) signals into alternating current (AC) or vice versa. There are two basic types of antenna: (1) The transmitting antenna, which is fed with $\mathrm{AC}$ from electronic equipments and generates an RF field, and (2) The receiving antenna, which intercepts RF energy and delivers AC to electronic equipment.

Antenna is three dimensional and lives in a world of beam area, steradians, square degree, and solid angle. It has self and mutual impedances. Antenna also has polarizations: linear, elliptical, and circular.

All types of antenna have the same basic principle that radiation is produced by accelerated (or decelerated) charge. The basic equation of radiation can be expressed as [1]:

$$
\dot{I} \iota=\mathrm{Qv}\left(\mathrm{Ams}^{-1}\right)
$$

where $\dot{\mathrm{I}}=$ time-changing current, As $^{-1}$

$\mathrm{L}=$ length of current element, $\mathrm{m}$

$$
\begin{aligned}
& \mathrm{Q}=\text { charge, } \mathrm{C} \\
& \mathrm{V}=\text { time change of velocity which equals the } \\
& \quad \text { acceleration of the charge, } \mathrm{ms}_{-2}
\end{aligned}
$$

Microstrip antennas are also known as printed antennas. There are several types of microstrip antennas in which the most common one is the patch antenna. A patch antenna is a narrowband, wide-beam antenna fabricated by etching the antenna element pattern in metal trace bonded to an insulating substrate.

Patch antennas incorporate the ability to have polarization diversity. With a single feed point, patch antennas can easily be designed to have vertical, horizontal, right hand circular polarization (RHCP) or left hand circular polarization (LHCP). This unique property allows antennas to be used in many areas of communication link with varied requirements. Patch antennas have a very low profile, are mechanically rugged and can be conformable. Hence, they are often mounted on the exterior of aircraft and spacecraft, and are incorporated into mobile radio communications devices.

Microstrip antennas are relatively inexpensive to manufacture and design due to the simple 2-dimensional physical geometry. They are usually utilized at ultra-high frequency (UHF) and higher frequencies because the size of the antenna is directly tied to the wavelength at the resonant frequency.

A fractal antenna is an antenna that uses a fractal design to maximize the length of material that transmits or receives electromagnetic signals within a given total surface area. Due to this reason, fractal antennas are very compact and hence are anticipated to have useful applications in cellular telephone and microwave communications.

Fractal antenna's response differs markedly from traditional antenna designs, in the sense that it is capable of operating optimally at many different frequency ranges simultaneously. This makes the fractal antenna an excellent design for broadband applications. 